\title{
The natural history of moderate aortic stenosis in a veteran population
}

\author{
Poornima Yechoor, BA, Alvin S. Blaustein, MD, Faisal G. Bakaeen, MD, Lorraine D. Cornwell, MD, \\ Joseph S. Coselli, MD, Scott A. LeMaire, MD, and Danny Chu, MD
} Objective: Our objective was to evaluate the natural history of moderate aortic stenosis in veterans-a unique
patient population with significant comorbidities.

\begin{abstract}
Methods: We retrospectively reviewed the records of all patients who underwent echocardiography at a single veterans affairs hospital during 2006. We identified consecutive patients who had moderate aortic stenosis as indicated by a mean transaortic gradient of 25 to $40 \mathrm{~mm} \mathrm{Hg}$, peak aortic jet velocity of 3 to $4 \mathrm{~m} / \mathrm{s}$, or aortic valve area of 1.0 to $1.5 \mathrm{~cm}^{2}$. The primary end point was defined as survival without aortic valve replacement.
\end{abstract}

Results: Of the 104 patients (mean age, $74 \pm 10$ years), $49 \%$ had diabetes, $21 \%$ had peripheral vascular disease, $21 \%$ were current smokers, $18 \%$ had chronic obstructive pulmonary disease, $60 \%$ had coronary artery disease, $89 \%$ had hypertension, and $31 \%$ had a body mass index of $30 \mathrm{~kg} / \mathrm{m}^{2}$ or more. Mean ejection fraction was $49 \% \pm 12 \%$. During the mean follow-up period of 22 months (range, $1-67$ months), $30 \%$ of patients underwent aortic valve replacement- $26 \%$ for symptomatic severe aortic stenosis and $4 \%$ concomitantly with coronary artery bypass grafting as the primary indicated operation-and $61 \%$ died. Event-free survivals were $48 \%$, $24 \%$, and $15 \%$ at 1,3 , and 5 years, respectively.

Conclusions: Our cohort of military veteran patients had significant comorbidities. Event-free survival for such patients who have moderate aortic stenosis is significantly lower than previously reported data suggest. Within this unique group of patients, identifying factors that accelerate the progression of moderate aortic stenosis would help surgeons select patients who may benefit from early aortic valve replacement for moderate aortic stenosis. (J Thorac Cardiovasc Surg 2013;145:1550-3)

Aortic stenosis (AS) is the most common type of valvular heart disease in the developed world. ${ }^{1-3}$ Calcific AS is a disease of the elderly, and as the population of the United States continues to age, the increasing prevalence of this type of AS represents a significant burden to patients and a topic of intense interest and study for physicians. ${ }^{1-4}$

The majority of the existing literature on the natural history of AS focuses on "severe" disease, and aortic valve replacement (AVR) has clearly been established as the standard of care in severe symptomatic AS. ${ }^{1,5}$ Among patients with "hemodynamically significant" AS per echocardiographic measurements, those who have angina,

From the Divisions of Cardiothoracic Surgery and Cardiology, Michael E. DeBakey Veterans Affairs Medical Center, Baylor College of Medicine, Texas Heart Institute at St Luke's Episcopal Hospital, Houston, Tex.

Funded by the Michael E. DeBakey Veterans Affairs Health Services Research \& Development Center of Excellence (HFP90-020); internal funding from Baylor College of Medicine.

Disclosures: Authors have nothing to disclose with regard to commercial support.

Presented at the 7th Annual Academic Surgical Congress, Las Vegas, Nevada, February 14-16, 2012.

Received for publication April 10, 2012; accepted for publication May 7, 2012; available ahead of print June 4, 2012.

Address for reprints: Danny Chu, MD, Divisions of Cardiothoracic Surgery, Texas Heart Institute at St Luke's Episcopal Hospital, Baylor College of Medicine, and the Michael E. DeBakey Veterans Affairs Medical Center, One Baylor Plaza, Houston, TX 77030 (E-mail: dchumd@gmail.com).

0022-5223/\$0.00

Published by Elsevier Inc. on behalf of The American Association for Thoracic Surgery

doi:10.1016/j.jtcvs.2012.05.013 syncope, dyspnea, or other symptoms of heart failure have been shown to have poorer survival and a greater risk of sudden death without prompt surgical intervention than patients with no symptoms., ${ }^{2,4,6-9}$

Asymptomatic moderate AS has been viewed as a relatively benign condition, and current practice guidelines recommend annual follow-up and surveillance echocardiography every 1 to 2 years for patients with moderate AS. ${ }^{1,4}$ Until recently, the natural history of moderate AS has been poorly defined. However, recent studies suggest that progression to severe disease can occur much more rapidly than previously expected and that moderate AS itself is associated with a substantial increase in mortality from both cardiac and noncardiac causes. ${ }^{10-13}$ The studies also identify characteristics (eg, moderate or severe aortic valve calcification, concomitant coronary artery disease [CAD]) that may put certain patients at risk for more rapid progression of their AS. ${ }^{13-15}$ These findings call into question the traditional paradigm of expectantly managing patients with moderate AS, suggesting instead that many patients require much closer follow-up and more aggressive management than the present guidelines indicate.

To date, the natural history of moderate AS has not been studied in the veteran population. Veterans represent a unique patient population with significant risk factors, including advanced age, concomitant $\mathrm{CAD}$, and other factors that have been shown to affect the progression of 


\section{Abbreviations and Acronyms \\ AS $\quad=$ aortic stenosis \\ AVR $\quad=$ aortic valve replacement \\ $\mathrm{CAD}=$ coronary artery disease \\ MEDVAMC $=$ Michael E. DeBakey Veterans \\ Affairs Medical Center}

AS. ${ }^{13-15}$ The aim of our study was to follow a cohort of military veterans with moderate AS to better understand the natural history and impact of this disease in this unique group of patients.

\section{PATIENTS AND METHODS \\ Patient Population}

The study was granted waiver of consent and approved by the Institutional Review Boards at the Michael E. DeBakey Veterans Affairs Medical Center (MEDVAMC) and Baylor College of Medicine, Houston, Tex. We retrospectively reviewed the medical records of all patients who underwent Doppler echocardiography at the MEDVAMC from January 1, 2006, to December 31, 2006. Patients with moderate AS were identified from the echocardiographic database maintained by the Division of Cardiology. For the purpose of our study, we defined moderate AS according to the current American College of Cardiology/American Heart Association valvular heart disease guidelines ${ }^{1}$ : a peak aortic jet velocity of 3.0 to $4.0 \mathrm{~m} / \mathrm{s}$, a mean transaortic valve pressure gradient of 25 to $40 \mathrm{~mm} \mathrm{Hg}$, or an aortic valve area of 1.0 to $1.5 \mathrm{~cm}^{2}$. We excluded patients who had previous AVR, incomplete echocardiographic data, or incomplete follow-up data.

We identified a total of 104 consecutive patients who met our prespecified criteria and conducted a detailed chart review, using the MEDVAMC Computerized Patient Record System, to extract pertinent demographic, echocardiographic, clinical, surgical, and follow-up data for each patient (Table 1). The presence of diabetes mellitus, hypertension, CAD, chronic obstructive pulmonary disease, and peripheral vascular disease as documented in the patients' charts was recorded. The degree of aortic valve calcification was not consistently documented in the echocardiographic reports reviewed and was thus not included in our study.

\section{Follow-up}

Follow-up data were available on the Computerized Patient Record System for all patients, and follow-up began from the date of the first echocardiogram that indicated moderate AS. The primary end point of our study was event-free survival, which was defined as freedom from death (from any cause) and AVR until the end of the follow-up period in August 2011.

\section{Statistical Analysis}

Continuous variables were expressed as mean \pm standard deviation. Probabilities of event-free survival at 1, 3, and 5 years were obtained by Kaplan-Meier estimates (STATA version 11; StataCorp LP, College Station, Tex).

\section{RESULTS}

\section{Event-Free Survival}

During the mean follow-up period of 22 months (range, 1-67 months), 89 patients died $(\mathrm{n}=58)$, underwent AVR $(\mathrm{n}=26)$, or underwent AVR and then died during the follow-up period $(\mathrm{n}=5)$. Estimated event-free survival was $48 \%$ at 1 year, $24 \%$ at 3 years, and $15 \%$ at 5 years (Figure 1).

\section{Aortic Valve Replacement}

Among the 31 patients who underwent AVR, severe symptomatic AS was the primary surgical indication in 27; the remaining 4 patients underwent AVR concomitantly with coronary artery bypass grafting because CAD was the primary indication for surgery. Of the 27 patients who had operations primarily for AVR, 15 had isolated AVR and 12 had concomitant coronary artery bypass grafting.

\section{Death}

Sixty-three patients died (from any cause) during the study period: 58 of the deaths were considered primary events and, as stated earlier, 5 deaths occurred after the patients underwent AVR. The available data did not allow us to record specific causes of death or whether deaths were of cardiac origin.

\section{Patients With No Events}

Of the $15(14 \%)$ patients in whom no events were observed, 3 had progressed to severe AS and 12 still had moderate AS at the end of the follow-up period. At that time, 10 of these patients were recommended for monitoring with surveillance echocardiography because of a lack of symptoms or disease progression. Two of the 15 patients were deemed not to be surgical candidates, 2 were recommended for AVR but wanted more time to decide, and 1 patient declined any further workup or intervention for his AS.

\section{DISCUSSION}

The current standard of care for severe symptomatic AS is AVR, because the existing data suggest that, otherwise, patients have an average survival of only 2 to 3 years after the onset of symptoms, and they have a high risk of sudden death. ${ }^{1,2,16-19}$ Current guidelines for the management of moderate AS recommend clinical follow-up yearly and echocardiography every 1 to 2 years. ${ }^{1,4}$ However, the results of our present study suggest that moderate AS may not be as benign as previously reported ${ }^{10,13}$ and that current practice guidelines may be inadequate, especially for the military veteran population.

Previous studies of the natural history of moderate AS have produced conflicting results. Specifically, Horstkotte and Loogen ${ }^{17}$ and Turina and associates ${ }^{18}$ suggest that moderate AS has a relatively benign course: Horstkotte and Loogen ${ }^{17}$ reported an average interval of 13.4 years from diagnosis to aortic valve surgery, and Turina's group ${ }^{18}$ reported $100 \%$ and $80 \%$ survivals at 1 and 4 years after diagnosis, respectively. However, in a small study, Kennedy and colleagues ${ }^{10}$ found that 14 of 66 patients died of causes attributed to moderate AS within 35 months after diagnosis. In addition, Otto's group ${ }^{12}$ showed that event-free survival 
TABLE 1. Patient characteristics $(N=104)$

\begin{tabular}{lc}
\hline \multicolumn{1}{c}{ Variable } & Value \\
\hline Age $(\mathrm{y})$ & $74 \pm 10$ \\
Gender (male) & $103(99 \%)$ \\
Body mass index $>30 \mathrm{~kg} / \mathrm{m}^{2}$ & $32(31 \%)$ \\
Current smoker & $22(21 \%)$ \\
Tobacco smoking history & $66(63 \%)$ \\
Diabetes mellitus & $51(49 \%)$ \\
Coronary artery disease & $62(60 \%)$ \\
Hypertension & $93(89 \%)$ \\
Chronic obstructive pulmonary disease & $19(18 \%)$ \\
Peripheral vascular disease & $22(21 \%)$ \\
Mean ejection fraction $(\%)$ & $49 \pm 12$ \\
Peak aortic jet velocity (m/s) & $3.2 \pm 0.6$ \\
Mean transaortic valve gradient (mm Hg) & $25.2 \pm 9.7$ \\
Aortic valve area $\left(\mathrm{cm}^{2}\right)$ & $1.1 \pm 0.3$ \\
\hline Data are presented as number (percentage) or as mean \pm standard deviation.
\end{tabular}

at 2 years was $66 \%$. Most recently, Rosenhek and associates ${ }^{13}$ reported $75 \%$ and $60 \%$ event-free survivals at 3 and 5 years, respectively. The same group also reported that patients with mild or moderate AS had an overall mortality that was $80 \%$ greater than that of age- and gendermatched controls.

Our results suggest that not only is the natural history of moderate AS in this veteran population not benign, but the event-free survival is significantly lower than even what Otto's ${ }^{12}$ and Rosenhek's groups ${ }^{13}$ had previously described. Eighty-five percent of patients had an event within our mean follow-up period of 22 months, and $61 \%$ of our patients died by the conclusion of our study, thus indicating that mortality in patients with moderate AS is not insignificant.

The body of literature on moderate AS also states that the rate of disease progression varies significantly among

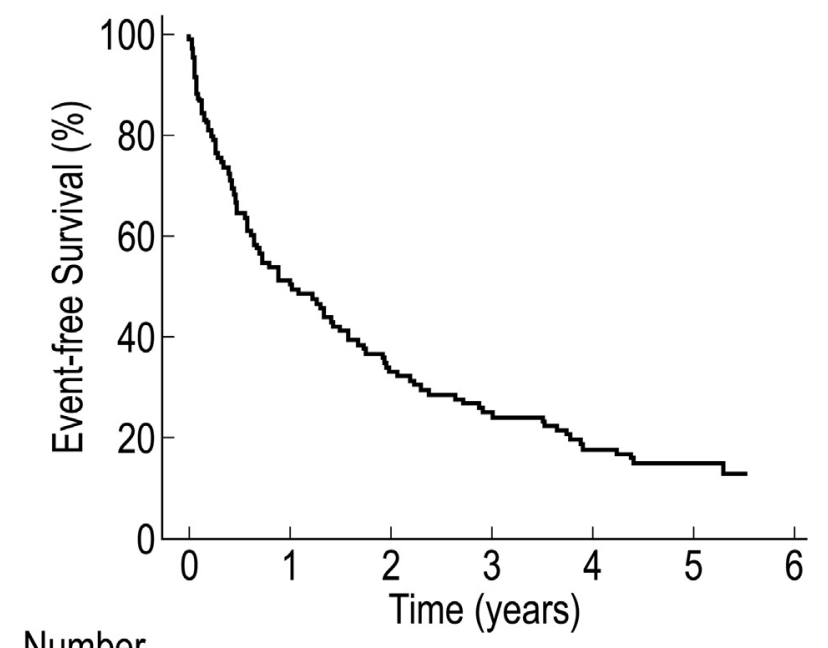

Number

$\begin{array}{llllllll}\text { at Risk } & 104 & 50 & 33 & 25 & 18 & 16 & 15\end{array}$

FIGURE 1. Kaplan-Meier event-free survival (events: aortic valve replacement, $\mathrm{n}=31$; death, $\mathrm{n}=58$ ). affected individuals. ${ }^{12,20}$ Some studies have attempted to identify risk factors for more rapid progression. 1,12-15,19,21 For example, studies have shown that severe disease develops more rapidly in patients with senile calcific stenosis than in those with rheumatic or congenital AS. ${ }^{15,19}$ Other factors that have been associated with the rapid progression of AS include advanced age, concomitant $\mathrm{CAD}$, and moderate to severe aortic valve calcification. ${ }^{13,15,19,21}$ Given that calcific AS is associated with aging, the association between advanced age and more rapid progression is not surprising. Similarly, with respect to its association with $\mathrm{CAD}$, calcific AS was previously understood to be a degenerative disease, but more recent studies suggest that the pathophysiology of calcific stenosis is in fact similar to that of atherosclerosis. $^{22,23}$ Thus, patients who have both AS and CAD probably have more severe aortic valve calcification than patients with AS alone. Finally, multiple studies have associated increased mortality with any degree of dystrophic calcification in either the aortic valve or the aorta. $^{24,25}$ In our cohort of veterans, patients tended to be older (average age, 74 years) and to have significant comorbidities. Of note, $60 \%$ of our patients had concomitant CAD.

As our population ages and health care costs continue to rise, the prevalence of AS will continue to increase, as well. Additionally, because the MEDVAMC provides cardiac care to veterans in a large geographic area (including southern Texas, Louisiana, Oklahoma, Arkansas, Mississippi, and western Florida), many of the military veterans who are seen at our facility have to travel great distances. Therefore, to aggressively follow up every patient with moderate AS documented by echocardiography may be logistically difficult. However, given that even within 1 year, eventfree survival is remarkably lower in this population than in other studied populations and that all-cause mortality in this group is high, we need to determine whether there is a subset of patients with moderate AS and a high risk of rapid disease progression who need to be followed up more closely than current guidelines indicate. The current literature suggests that more attention must be paid to patients with concomitant CAD and that the severity of aortic valve calcification should be recognized as an important predictor of outcome. Studying patients with these characteristics and identifying any other pertinent predictors unique to the veteran population will be an important next step, and modifying the existing guidelines for more vulnerable patients and involving both cardiologists and cardiac surgeons early on in these cases will allow the management of moderate AS to be tailored to individual patients' needs.

Our study is subject to the limitations inherent in a retrospective review. Because our patient cohort was limited to military veterans, nearly all of our patients were men. Consequently, we cannot generalize our results to female 
patients. Also, whereas we carefully reviewed each echocardiographic report to verify the severity of each patient's AS, we did not view the actual echocardiographic images to verify the measurements. Thus, the parameters used in our analyses were dependent on the image quality of the echocardiogram and on the judgment of the interpreting cardiologist. Additionally, the concomitant presence of symptoms of interest, including angina, syncope, and dyspnea, could not be determined for our patients at the time of each echocardiographic examination because of inconsistencies within their medical records.

\section{CONCLUSIONS}

Our study is significant not only because it corroborates emerging evidence that moderate AS is not a benign condition, but also because it focuses on the unique population of military veterans, who have significant comorbidities and risk factors. We found that moderate AS in this population is associated with rapid progression and considerable mortality. Therefore, the current practice guidelines for the management of moderate AS may not be appropriate for this group of patients. Further study of other prognostic factors unique to the veteran population is needed to improve our ability to assess and manage this disease in an individualized manner.

Stephen N. Palmer, PhD, ELS, contributed to the editing of the manuscript.

\section{References}

1. Bonow RO, Carabello BA, Chatterjee K, de Leon AC Jr, Faxon DP, Freed MD, et al. 2008 Focused update incorporated into the ACC/AHA 2006 guidelines for the management of patients with valvular heart disease: a report of the American College of Cardiology/American Heart Association Task Force on Practice Guidelines (Writing Committee to Revise the 1998 Guidelines for the Management of Patients With Valvular Heart Disease): endorsed by the Society of Cardiovascular Anesthesiologists, Society for Cardiovascular Angiography and Interventions, and Society of Thoracic Surgeons. Circulation. 2008;118: e523-661.

2. Lester SJ, Heilbron B, Gin K, Dodek A, Jue J. The natural history and rate of progression of aortic stenosis. Chest. 1998;113:1109-14.

3. Supino PG, Borer JS, Yin A, Dillingham E, McClymont W. The epidemiology of valvular heart diseases: the problem is growing. Adv Cardiol. 2004;41:9-15.
4. Vahanian A, Baumgartner H, Bax J, Butchart E, Dion R, Filippatos G, et al. Guidelines on the management of valvular heart disease: The Task Force on the Management of Valvular Heart Disease of the European Society of Cardiology. Eur Heart J. 2007;28:230-68.

5. Ross J Jr, Braunwald E. Aortic stenosis. Circulation. 1968;38:61-7.

6. Carabello BA. Timing of valve replacement in aortic stenosis: moving closer to perfection. Circulation. 1997;95:2241-3.

7. Carabello BA. Evaluation and management of patients with aortic stenosis. Circulation. 2002;105:1746-50.

8. Carabello BA, Paulus WJ. Aortic stenosis. Lancet. 2009;373:956-66

9. Otto CM. Valvular aortic stenosis: disease severity and timing of intervention. J Am Coll Cardiol. 2006;47:2141-51.

10. Kennedy KD, Nishimura RA, Holmes DR Jr, Bailey KR. Natural history of moderate aortic stenosis. J Am Coll Cardiol. 1991;17:313-9.

11. Otto CM. Aortic stenosis: even mild disease is significant. Eur Heart J. 2004;25 185-7.

12. Otto CM, Burwash IG, Legget ME, Munt BI, Fujioka M, Healy NL, et al Prospective study of asymptomatic valvular aortic stenosis: clinical, echocardiographic, and exercise predictors of outcome. Circulation. 1997;95: 2262-70.

13. Rosenhek R, Klaar U, Schemper M, Scholten C, Heger M, Gabriel H, et al. Mild and moderate aortic stenosis: natural history and risk stratification by echocardiography. Eur Heart J. 2004;25:199-205.

14. Bahler RC, Desser DR, Finkelhor RS, Brener SJ, Youssefi M. Factors leading to progression of valvular aortic stenosis. Am J Cardiol. 1999;84:1044-8.

15. Peter M, Hoffmann A, Parker C, Luscher T, Burckhardt D. Progression of aortic stenosis: role of age and concomitant coronary artery disease. Chest. 1993;103: 1715-9.

16. Frank S, Johnson A, Ross J Jr. Natural history of valvular aortic stenosis Br Heart J. 1973;35:41-6.

17. Horstkotte D, Loogen F. The natural history of aortic valve stenosis. Eur Heart J. 1988;9 Suppl E:57-64.

18. Turina J, Hess O, Sepulcri F, Krayenbuehl HP. Spontaneous course of aortic valve disease. Eur Heart J. 1987;8:471-83.

19. Wagner S, Selzer A. Patterns of progression of aortic stenosis: a longitudinal hemodynamic study. Circulation. 1982;65:709-12.

20. Freeman RV, Otto CM. Spectrum of calcific aortic valve disease: pathogenesis, disease progression, and treatment strategies. Circulation. 2005;111:3316-26.

21. Palta S, Pai AM, Gill KS, Pai RG. New insights into the progression of aortic stenosis: implications for secondary prevention. Circulation. 2000;101: 2497-502.

22. Otto CM, Kuusisto J, Reichenbach DD, Gown AM, O'Brien KD. Characterization of the early lesion of 'degenerative' valvular aortic stenosis: histological and immunohistochemical studies. Circulation. 1994;90:844-53.

23. Rajamannan NM, Gersh B, Bonow RO. Calcific aortic stenosis: from bench to the bedside-emerging clinical and cellular concepts. Heart. 2003;89: $801-5$.

24. Otto CM, Lind BK, Kitzman DW, Gersh BJ, Siscovick DS. Association of aorticvalve sclerosis with cardiovascular mortality and morbidity in the elderly. $N$ Engl J Med. 1999;341:142-7.

25. Witteman JC, Kok FJ, van Saase JL, Valkenburg HA. Aortic calcification as a predictor of cardiovascular mortality. Lancet. 1986;2:1120-2. 